\title{
Long range rapidity correlations as seen in the STAR experiment
}

\author{
T. Lappi ${ }^{\mathrm{a}, \mathrm{b}}$, L. McLerran ${ }^{\mathrm{c}}$ \\ ${ }^{a}$ Department of Physics, P.O. Box 35, 40014 University of Jyväskylä, Finland \\ ${ }^{b}$ Institut de Physique Théorique, Bât. 774, CEA/DSM/Saclay, 91191 Gif-sur-Yvette, France \\ ${ }^{c}$ Physics Department and Riken-BNL Center, Brookhaven National Laboratory, Upton, NY 11973, USA
}

\begin{abstract}
We analyze long range rapidity correlations observed in the STAR experiment at RHIC. Our goal is to extract properties of the two particle correlation matrix, accounting for the analysis method of the STAR experiment. We find a surprisingly large correlation strength for central collisions of gold nuclei at highest RHIC energies. We argue that such correlations cannot be the result of impact parameter fluctuations.
\end{abstract}

Key words:

\section{Introduction}

The STAR experiment at RHIC has reported preliminary observations of forward backward correlations as a function of the centrality of the collision [1, 2]. The reported forward-backward correlation shows a rapid increase in strength as a function of centrality, and appears to have a strength which cannot be explained by a superposition of $p p$ interactions. It has been suggested that such correlations are a consequence of the Color Glass Condensate induced Glasma produced in the early stages of heavy ion collisions $3,4,45,6,7,8,19,10,11,12,13,14,15,16,17,18,19,20]$ An alternative but quite similar explanation is provided by parton percolation [21, 22, 23]. The long range correlations may be the origin of the ridge phenomenon measured at RHIC, 224, 25, 26, 27, 28, 29, 30, 31 that may also be explained as arising from color electric and magnetic flux tubes originating in the Glasma [32, 33, 34, 35].

The results for the forward-backward correlations strength measured in the STAR experiment are reported as a function of centrality bin. One might be worried that if one averages over all the events in a centrality bin, correlations would be generated by the different impact parameters (or numbers of wounded nucleons) possible within such a bin; this is essentially the mechanism proposed in [36, 37, 38, 39]. We expect the charged multiplicity to be strongly correlated with impact parameter and if the impact parameter itself can have significant variation within a fixed centrality bin, then spurious correlations whose only origin is the geometry of the collision would be generated.

The STAR analysis is however more sophisticated. For each event one measures also a reference multiplicity in a relatively wide central rapidity interval. Correlations and fluctuations of the forward and backward multiplicities are then measured separately for each reference multiplicity. The correlations and fluctuations reported are average values of these measurements over each centrality bin. One might expect that such a procedure is relatively insensitive to impact parameter correlations.

Preprint submitted to Elsevier 
One purpose of this paper is to show how to extract values of the multiplicity correlation function including the constraints actually applied by the the STAR experiment. After setting up some notations in sec. 2 we argue in sec. 3 that, within a Gaussian approximation for the fluctuations, there is a bound on the ratio of forward backward correlated fluctuations to forward fluctuations,

$$
b=\frac{\left\langle N_{\mathrm{F}} N_{\mathrm{B}}\right\rangle_{N_{\mathrm{R}}}-\left\langle N_{\mathrm{F}}\right\rangle_{N_{\mathrm{R}}}\left\langle N_{\mathrm{B}}\right\rangle_{N_{\mathrm{R}}}}{\left\langle N_{\mathrm{F}}^{2}\right\rangle_{N_{\mathrm{R}}}-\left\langle N_{\mathrm{F}}\right\rangle_{N_{\mathrm{R}}}^{2}} .
$$

Here, $N_{\mathrm{F}}$ is the multiplicity measured in some forward bin of rapidity, and $N_{\mathrm{B}}$ the value in a backwards bin, and the subscript $N_{\mathrm{R}}$ indicates that the averages are taken for fixed values of the reference multiplicity. In the STAR experiment the pseudorapidity windows are at symmetric values of pseudorapidity around $\eta=0$. When the forward and backward rapidity windows are smaller than the reference window and farther from each other than from the reference window, we argue on quite general grounds that $b<1 / 2$. This is perhaps violated by non-linear effects that are of order $1 /\left\langle N_{\mathrm{F}}\right\rangle$, effects that could be several percent for the most central collisions. If $b=1 / 2$, the multiplicities are maximally correlated. This bound is derived making, besides this Gaussian approximation, only minimal assumptions about the correlations and does not depend on the detailed mechanism for generating them. The STAR data is consistent with this bound except for the most central events of $\mathrm{Au}-\mathrm{Au}$ collisions at the highest beam energy, where there are small violations. We then analyse the effect of the impact parameter induced correlations of the type suggested in Ref. [36, 37, 38, 39] in sec. 4 and find that they are not sufficient to explain the large correlation seen in the STAR data. In sec. 5 we then propose a simple parametrization to add intrinsic long range correlations to the impact parameter fluctuations.

Our results are: The correlations measured in STAR do not appear to be entirely associated with impact parameter fluctuations. The measured value of $b$ is near its maximally allowed value for central $\mathrm{Au}$-Au collisions at RHIC energy. Indeed for the highest energy and most central $\mathrm{Au}-\mathrm{Au}$ collisions it is larger than the bound $b<1 / 2$. We find that a crucial requirement to obtain a $b$ close to this limit are large fluctuations in the reference multiplicity.

\section{The rapidity correlation function}

The quantity that we wish to extract information from experiment is the rapidity correlation function. We define it as

$$
C\left(\eta, \eta^{\prime}\right) \equiv\left\langle\frac{\mathrm{d} N}{\mathrm{~d} \eta} \frac{\mathrm{d} N}{\mathrm{~d} \eta^{\prime}}\right\rangle-\left\langle\frac{\mathrm{d} N}{\mathrm{~d} \eta}\right\rangle\left\langle\frac{\mathrm{d} N}{\mathrm{~d} \eta^{\prime}}\right\rangle
$$

This can be decomposed into a local piece that corresponds to Poissonian fluctuations in the particle number and a long range correlaion

$$
C\left(\eta-\eta^{\prime}\right)=\delta\left(\eta-\eta^{\prime}\right)\left\langle\frac{\mathrm{d} N}{\mathrm{~d} \eta}\right\rangle+K\left(\eta-\eta^{\prime}\right)\left\langle\frac{\mathrm{d} N}{\mathrm{~d} \eta}\right\rangle\left\langle\frac{\mathrm{d} N}{\mathrm{~d} \eta^{\prime}}\right\rangle .
$$

The $\delta\left(\eta-\eta^{\prime}\right)$ piece is often (e.g. [40, 41]) absorbed into the definition of the correlation function itself (the l.h.s. of eq. (2) ), but it will be convenient for the following discussion to keep it on the r.h.s. as part of $C\left(\eta-\eta^{\prime}\right)$.

Some information about the function $K$ can be found by measuring local multiplicity fluctuations. Integrating eq. (3) twice over a pseudorapidity interval $\Delta \eta$ centered at some rapidity $\eta$ and 
assuming that $\Delta \eta$ is sufficiently small that the pseudorapidity dependence of the multiplicity in this interval can be neglected, we obtain

$$
\left\langle n^{2}\right\rangle-\langle n\rangle^{2}=\langle n\rangle+\frac{\langle n\rangle^{2}}{k} .
$$

This result is identical to the result for obtained a negative binomial distribution, and we can identify the $k$-parameter as

$$
\frac{1}{k}=\int_{\eta-\Delta \eta / 2}^{\eta+\Delta \eta / 2} \mathrm{~d} \eta \mathrm{d} \eta^{\prime} K\left(\eta-\eta^{\prime}\right) .
$$

(The negative binomial distribution has further consequences for higher moments of the multiplicity distribution as well.) This relation can be used to gain information on the rapidity correlation for small rapidity differences from measurements of the multiplicity distribution in different $\Delta \eta$ intervals [40, 41]

Of central interest in this paper is the behavior of the correlation function $C$ at large rapidity differences. In particular, we shall concentrate on the case studied by the STAR collaboration [1, 2]. This analysis measures the correlation between charged particle multiplicities in forward ("F") and backward ("B") rapidities. Specifically, pseudo-rapidity windows of width $\delta=0.2$, are situated symmetrically around midrapidity within the STAR TPC acceptance $|\eta|<1$. The data is divided into $10 \%$ centrality bins and the correlation coefficient between the forward and backward multiplicities is measured within the centrality bin. In order to eliminate the effect of centrality fluctuations within one bin, there is an additional crucial twist in the analysis. One also measures the multiplicity in a third reference ("R") rapidity interval of width $\delta_{\mathrm{R}}=1.0$ that does not overlap with the "F" and "B" windows. The variances and the covariance of the " $\mathrm{F}$ " and "B" windows are then determined separately for each reference multiplicity $N_{\mathrm{R}}$, the motivation being that a fixed $N_{\mathrm{R}}$ selects, with a good accuracy, events with a fixed impact parameter. Although the data is presented for rapidity intervals in different locations, it is good to keep in mind a typical configuration, with rapidity windows $-1<\eta<-0.8$ ("B"), $-0.5<\eta<0.5$ ("R") and $0.8<\eta<1$ ("F").

\section{Gaussian approximation}

As we have discussed, in order to analyze the STAR results, we shall have to study the case of three correlated multiplicities, $N_{\mathrm{F}}, N_{\mathrm{B}}$ and $N_{\mathrm{R}}$. We shall first compute such fluctuations in a Gaussian approximation for the probability distribution of these three variables within all the events in a centrality bin. This should be a good approximation when considering fluctuations around an average multiplicity, so long as that average multiplicity is large. This is a consequence of the central limit theorem, and in sec. 4 we shall see explicitly how it follows directly from a stationary phase approximation to expressions for the multiplicity fluctuations in a simple model. The corrections to this approximations should be of order $1 / N$, where $N$ is a multiplicity in a bin. For the STAR experiment, and central events, this should be valid to a few percent, since the typical value of $N_{F, B} \sim 100$ for the forward or backward multiplicity. However, in events with centrality around $50 \%, N_{F, B} \sim 20$, so one can expect $10-20 \%$ corrections.

We assume that there is some requirement on the centrality of the collision that fixes the average values $\left\langle N_{\mathrm{F}}\right\rangle,\left\langle N_{\mathrm{B}}\right\rangle$, and $\left\langle N_{\mathrm{R}}\right\rangle$. We define $\Delta_{U}=N_{U}-\left\langle N_{U}\right\rangle$, where $U$ is $\mathrm{F}$, B or $\mathrm{R}$, so that the 
normalized probability distribution is

$$
P\left(N_{\mathrm{F}}, N_{\mathrm{B}} ; N_{\mathrm{R}}\right)=\frac{1}{(2 \pi)^{3 / 2} \operatorname{det} \Sigma} \exp \left[-\frac{1}{2} \Delta_{U} \Sigma_{U V}^{-1} \Delta_{V}\right]
$$

In this equation

$$
\Sigma_{U V} \equiv \sigma_{U V}^{2}=\left\langle\Delta_{U} \Delta_{V}\right\rangle=\int_{U} \mathrm{~d} \eta \int_{V} \mathrm{~d} \eta^{\prime} C\left(\eta-\eta^{\prime}\right) \quad, \quad U, V=\mathrm{F}, \mathrm{B}, \mathrm{R} .
$$

In all that follows, we will assume that the forward and backward windows are chosen to be centered at rapidity values symmetric around $\eta=0$, and that the reference multiplicity is chosen by summing over rapidity values that are also symmetrically displaced. Since $\Sigma_{U V}=\Sigma_{V U}$ by construction this means that

$$
\begin{aligned}
\Sigma_{\mathrm{FB}} & =\Sigma_{\mathrm{BF}} \\
\Sigma_{\mathrm{FR}}= & \Sigma_{\mathrm{BR}}=\Sigma_{\mathrm{BR}}=\Sigma_{\mathrm{RB}}
\end{aligned}
$$

Other than the Gaussian form and the symmetry above, we shall in this section make no assumptions about the physical origin or strength of the correlation; except that it is a decreasing function of the rapidity separation between the measured multiplicities. We also introduce the correlation coefficient between two multiplicities with the conventional definition

$$
R_{U V}=\frac{\Sigma_{U V}}{\sqrt{\Sigma_{U U} \Sigma_{V V}}}
$$

The correlation coefficient is mathematically restricted (due to the Cauchy-Schwarz inequality) to values between -1 and 1 . Without fixing the reference multiplicity, the covariance $D_{b f}$, variance $D_{f f}$ and correlation coefficient $b$ used in [1, 2] would be the same as $\sigma_{\mathrm{FB}}^{2} \sigma_{\mathrm{FF}}^{2}$ and $R_{\mathrm{BF}}$. We shall however reserve the notation $D_{b f} D_{f f}$ and $b$ to the quantities at fixed $N_{\mathrm{R}}$ that are actually measured. The probability distribution (6) involves $\Sigma_{U V}^{-1}$, the inverse of the correlation matrix $\Sigma$. As a $3 \times 3$ matrix $\Sigma$ is easily inverted to give

$$
\Sigma^{-1}=\frac{1}{\operatorname{det} \Sigma}\left(\begin{array}{ccc}
\sigma_{\mathrm{BB}}^{2} \sigma_{\mathrm{RR}}^{2}-\left(\sigma_{\mathrm{BR}}^{2}\right)^{2} & \sigma_{\mathrm{BR}}^{2} \sigma_{\mathrm{FR}}^{2}-\sigma_{\mathrm{FB}}^{2} \sigma_{\mathrm{RR}}^{2} & \sigma_{\mathrm{BR}}^{2} \sigma_{\mathrm{FB}}^{2}-\sigma_{\mathrm{BB}}^{2} \sigma_{\mathrm{FR}}^{2} \\
\sigma_{\mathrm{BR}}^{2} \sigma_{\mathrm{FR}}^{2}-\sigma_{\mathrm{FB}}^{2} \sigma_{\mathrm{RR}}^{2} & \sigma_{\mathrm{FF}}^{2} \sigma_{\mathrm{RR}}^{2}-\left(\sigma_{\mathrm{FR}}^{2}\right)^{2} & \sigma_{\mathrm{FB}}^{2} \sigma_{\mathrm{FR}}^{2}-\sigma_{\mathrm{BR}}^{2} \sigma_{\mathrm{FF}}^{2} \\
\sigma_{\mathrm{BR}}^{2} \sigma_{\mathrm{FB}}^{2}-\sigma_{\mathrm{BB}}^{2} \sigma_{\mathrm{FR}}^{2} & \sigma_{\mathrm{FB}}^{2} \sigma_{\mathrm{FR}}^{2}-\sigma_{\mathrm{BR}}^{2} \sigma_{\mathrm{FF}}^{2} & \sigma_{\mathrm{BB}}^{2} \sigma_{\mathrm{FF}}^{2}-\left(\sigma_{\mathrm{FB}}^{2}\right)^{2}
\end{array}\right) .
$$

When the reference multiplicity is not measured, the probability distribution can be reduced to the double distribution for $\Delta_{\mathrm{F}}, \Delta_{\mathrm{B}}$ by integrating over $\Delta_{\mathrm{R}}$

$$
\begin{aligned}
P\left(\Delta_{\mathrm{F}}, \Delta_{\mathrm{B}}\right) & =\int \mathrm{d} \Delta_{\mathrm{R}} P\left(\Delta_{\mathrm{F}}, \Delta_{\mathrm{B}}, \Delta_{\mathrm{R}}\right) \\
& =\frac{1}{(2 \pi)^{3 / 2} \operatorname{det} \Sigma_{2}} \exp \left[-\frac{\Delta_{U} \Sigma_{2}^{-1}{ }_{U V} \Delta_{V}}{2}\right], \quad U, V=F, B
\end{aligned}
$$

where $\Sigma_{2}^{-1}$ is the inverse of the $2 \times 2$ correlation matrix

$$
\Sigma_{2}=\left(\begin{array}{cc}
\sigma_{\mathrm{FF}}^{2} & \sigma_{\mathrm{FB}}^{2} \\
\sigma_{\mathrm{FB}}^{2} & \sigma_{\mathrm{BB}}^{2}
\end{array}\right) .
$$


In our case, however, we want the probability distribution of $\Delta_{F}, \Delta_{B}$ for a fixed $\Delta_{R}$, not integrated over all values of $\Delta_{R}$. In this case the (conditional on $\Delta_{R}$ ) probability distribution for $\Delta_{F}, \Delta_{B}$ is given by

$$
\begin{aligned}
& P\left(\Delta_{\mathrm{F}}, \Delta_{\mathrm{B}} \mid \Delta_{\mathrm{R}}\right)=\frac{P\left(\Delta_{\mathrm{F}}, \Delta_{\mathrm{B}}, \Delta_{\mathrm{R}}\right)}{P\left(\Delta_{\mathrm{R}}\right)} \\
&=\frac{1}{2 \pi \operatorname{det} \widetilde{\Sigma}} \exp \left[-\frac{\Delta_{U} \widetilde{\Sigma}_{U V}^{-1} \Delta_{V}}{2}-\Sigma^{-1}{ }_{U \mathrm{R}} \Delta_{U} \Delta_{\mathrm{R}}-\frac{\Sigma^{-1} \mathrm{RR}-\left(\Sigma_{\mathrm{RR}}\right)^{-1}}{2} \Delta_{\mathrm{R}}^{2}\right] \\
& \quad U, V=\mathrm{F}, \mathrm{B} .
\end{aligned}
$$

The presence of mixed terms in $\Delta_{F} \Delta_{R}, \Delta_{B} \Delta_{R}$ means that the expectation values of $\Delta_{F}, \Delta_{B}$ are shifted from zero and proportional to $\Delta_{\mathrm{R}}$. This is natural, since if one has an event with exceptionally large $N_{\mathrm{R}}$ one also expects a large $N_{\mathrm{F}}$ and $N_{\mathrm{B}}$ because the multiplicities are correlated. The term in $\Delta_{\mathrm{R}}^{2}$ assures the correct normalization $\int \mathrm{d} \Delta_{\mathrm{F}} \mathrm{d} \Delta_{\mathrm{B}} P_{\mathrm{R}}\left(\Delta_{\mathrm{F}}, \Delta_{\mathrm{B}}\right)=1$. The fluctuations and correlations of $\Delta_{\mathrm{F}}, \Delta_{\mathrm{B}}$ are determined by the coefficients of the quadratic part. They are described by the reduced correlation matrix $\widetilde{\Sigma}$, obtained by taking the F, B-elements of $\Sigma^{-1}$ from eq. (11)

$$
\widetilde{\Sigma}^{-1}=\frac{1}{\operatorname{det} \Sigma}\left(\begin{array}{cc}
\sigma_{\mathrm{BB}}^{2} \sigma_{\mathrm{RR}}^{2}-\left(\sigma_{\mathrm{BR}}^{2}\right)^{2} & \sigma_{\mathrm{BR}}^{2} \sigma_{\mathrm{FR}}^{2}-\sigma_{\mathrm{FB}}^{2} \sigma_{\mathrm{RR}}^{2} \\
\sigma_{\mathrm{BR}}^{2} \sigma_{\mathrm{FR}}^{2}-\sigma_{\mathrm{FB}}^{2} \sigma_{\mathrm{RR}}^{2} & \sigma_{\mathrm{FF}}^{2} \sigma_{\mathrm{RR}}^{2}-\left(\sigma_{\mathrm{FR}}^{2}\right)^{2}
\end{array}\right) .
$$

To get the correlation matrix of $\Delta_{\mathrm{F}}, \Delta_{\mathrm{B}}$ for a fixed $\Delta_{\mathrm{R}}$ we must then again invert this matrix to get

$$
\widetilde{\Sigma}=\left(\begin{array}{cc}
\sigma_{\mathrm{FF}}^{2}-\frac{\left(\sigma_{\mathrm{FR}}^{2}\right)^{2}}{\sigma_{\mathrm{RR}}^{2}} & \sigma_{\mathrm{FB}}^{2}-\frac{\sigma_{\mathrm{BR}}^{2} \sigma_{\mathrm{FR}}^{2}}{\sigma_{\mathrm{RR}}^{2}} \\
\sigma_{\mathrm{FB}}^{2}-\frac{\sigma_{\mathrm{BR}}^{2} \sigma_{\mathrm{FR}}^{2}}{\sigma_{\mathrm{RR}}^{2}} & \sigma_{\mathrm{BB}}^{2}-\frac{\left(\sigma_{\mathrm{BR}}^{2}\right)^{2}}{\sigma_{\mathrm{RR}}^{2}}
\end{array}\right) .
$$

From this we can read off correlation coefficients that correspond to the measured observables

$$
\begin{aligned}
D_{f f} & =\widetilde{\Sigma}_{\mathrm{FF}}=\sigma_{\mathrm{FF}}^{2}-\frac{\left(\sigma_{\mathrm{FR}}^{2}\right)^{2}}{\sigma_{\mathrm{RR}}^{2}} \\
D_{b f} & =\widetilde{\Sigma}_{\mathrm{BF}}=\sigma_{\mathrm{FB}}^{2}-\frac{\sigma_{\mathrm{BR}}^{2} \sigma_{\mathrm{FR}}^{2}}{\sigma_{\mathrm{RR}}^{2}} \\
b & =\frac{D_{b f}}{\sqrt{D_{f f} D_{b b}}}=\frac{R_{\mathrm{BF}}-R_{\mathrm{BR}} R_{\mathrm{FR}}}{\sqrt{\left(1-\left(R_{\mathrm{BR}}\right)^{2}\right)\left(1-\left(R_{\mathrm{FR}}\right)^{2}\right)}} .
\end{aligned}
$$

The shifts in the expectation values of the $\Delta$ 's for a fixed reference multiplicity can be computed in terms of the matrix elements of $\Sigma$ as

$$
\left\langle\Delta_{\mathrm{F}, \mathrm{B}}\right\rangle_{N_{\mathrm{R}}}=\frac{\sigma_{\mathrm{FR}}^{2}}{\sigma_{\mathrm{RR}}^{2}} \Delta_{\mathrm{R}}
$$

The information in the correlation between the reference multiplicity and the forward and backward multiplicity, as well as the fluctuations in the reference multiplicity itself contain some non-trivial information about the correlations. 
Equation (19) is the central result of this section. It describes how measurement of the forwardbackward correlation is modified by the fact that the multiplicities are observed for a fixed reference multiplicity, which in turn is correlated with the forward and backward multiplicities. We shall now turn to analysing its consequences using some very general assumptions on the two particle correlation (2). The corrections caused by the fixed reference multiplicity have a very intuitive meaning. For a fixed reference multiplicity the $\Delta_{\mathrm{F}, \mathrm{B}}$ fluctuate less, $\widetilde{\Sigma}_{\mathrm{FF}}<\sigma_{\mathrm{FF}}^{2}$. This is due to the correlation between the reference and F,B multiplicities and the effect goes away when $R_{\mathrm{FR}} \rightarrow 0$. The modification also decreases when the reference multiplicity has larger (uncorrelated) fluctuations $\sigma_{\mathrm{RR}}^{2}$.

In the limit of very strong correlations, the correlation coefficients $R$ are all very large. This is the case in heavy ion collisions when the centrality bins are taken to be very wide so that fluctuations and correlations are dominated by impact parameter fluctuations within the centrality bin. Let us first assume, for simplicity, that all the correlation coefficients are equal, $R_{\mathrm{BF}}=R_{\mathrm{FR}}=R_{\mathrm{BR}}=R$. Now the conditional correlation coefficient (19) is given by

$$
b=\frac{R-R^{2}}{1-R^{2}}=\frac{R}{1+R} .
$$

Remembering that $R \leq 1$ this lead to the upper limit $b<1 / 2$.

Consider now the typical experimental situation mentioned above, with rapidity windows $-1<$ $\eta<-0.8$ ("B"), $-0.5<\eta<0.5$ ("R") and $0.8<\eta<1$ ("F"). We shall only assume that the correlation function $C\left(\eta, \eta^{\prime}\right)$ is boost-invariant and symmetric, i.e. a function of $\left|\eta-\eta^{\prime}\right|$ only. Any reasonable correlation function $C\left(\left|\eta-\eta^{\prime}\right|\right)$ will decrease as a function of $\left|\eta-\eta^{\prime}\right|$. These very general assumptions inserted into eq. (7) lead to simple inequalities for the correlation matrix:

$$
\frac{1}{\delta_{\mathrm{R}}^{2}} \sigma_{\mathrm{RR}}^{2} \leq \frac{1}{\delta^{2}} \sigma_{\mathrm{FF}}^{2}=\frac{1}{\delta^{2}} \sigma_{\mathrm{BB}}^{2} \text { for } \delta_{\mathrm{R}}>\delta .
$$

Because in our typical configuration the $\mathrm{F}$, B-windows are closer to the reference rapidity window than to each other, the average value of the correlation function between the $\mathrm{F}, \mathrm{B}$ windows and the reference window is also larger. This leads to the estimate

$$
\frac{1}{\delta_{\mathrm{R}} \delta} \sigma_{\mathrm{FR}}^{2} \geq \frac{1}{\delta^{2}} \sigma_{\mathrm{FB}}^{2}
$$

Together eqs. (22) and (23) imply that $R_{\mathrm{FR}}=R_{\mathrm{BR}} \geq R_{\mathrm{BF}}$. Because the constrained correlation coefficient $b$ in eq. (19) is a monotonously decreasing function of $R_{\mathrm{FR}}=R_{\mathrm{BR}}$, we then get the upper limit

$$
b=\frac{R_{\mathrm{BF}}-R_{\mathrm{FR}}^{2}}{1-R_{\mathrm{FR}}^{2}} \leq \frac{R_{\mathrm{BF}}-R_{\mathrm{BF}}^{2}}{1-R_{\mathrm{BF}}^{2}}=\frac{R_{\mathrm{BF}}}{1+R_{\mathrm{BF}}} \leq 0.5 \text { for } \delta_{\mathrm{R}}>\delta .
$$

The preliminary STAR results [1, 2, 42] violate this bound derived from very general assumptions, and at the moment we see no compelling explanation for this discrepancy. Note also that for the configurations where the F, B-windows are close to each other, their distance from each other can be less than that to the reference rapidity region. When F, B windows are closer than 0.6 units in rapidity, the reference window is determined as $0.5<|\eta|<1.0$ and eq. (23) no longer strictly applies. In this region the observed value of $b$ does indeed tend to be slightly larger than for large rapidity separations. 


\section{Poissonian and impact parameter fluctuation model}

\subsection{The model}

For an illustration let us consider the toy model introduced in Ref. [36], where the authors claim that the impact parameter fluctuations within the centrality bin would explain most of the STAR correlation measurement. The model includes a short range Poisson correlation and the fluctuation of the collision geometry via a Monte Carlo Glauber (MCG) model. In practice the charged particles produced in a nucleus-nucleus collision are assumed to be a superposition of particle production from each participant nucleon:

$$
N_{\text {ch }}=\sum_{i=1}^{N_{\mathrm{p}}} n_{i}
$$

where $N_{\mathrm{p}}$ is the number of participant nucleons. The variables $n_{i}$ are independent and distributed according to a Poisson distribution with mean $\bar{n}$. These particles are then distributed in pseudorapidity with a gaussian distribution so that

$$
\left\langle\frac{\mathrm{d} N}{\mathrm{~d} \eta}\right\rangle=\left\langle N_{\mathrm{p}}\right\rangle \frac{\bar{n}}{\sqrt{2 \pi \sigma_{\eta}}} e^{-\frac{\eta^{2}}{2 \sigma_{\eta}^{2}}} .
$$

\subsection{Gaussian approximation}

Knowing that the only correlations in this toy model are the local one leading to the Poisson distribution and an infinite range one from the fluctuating number of participant nucleons, one can immediately write down 11 the correlation function of the model:

$$
C\left(\eta, \eta^{\prime}\right)=\delta\left(\eta-\eta^{\prime}\right)\left\langle\frac{\mathrm{d} N}{\mathrm{~d} \eta}\right\rangle+\frac{\sigma_{\mathrm{p}}^{2}}{\left\langle N_{\mathrm{p}}\right\rangle^{2}}\left\langle\frac{\mathrm{d} N}{\mathrm{~d} \eta}\right\rangle\left\langle\frac{\mathrm{d} N}{\mathrm{~d} \eta^{\prime}}\right\rangle,
$$

where $\sigma_{\mathrm{p}}^{2}$ is the variance of the number of participants in the given centrality bin. It is easy to see that this correlation function, when integrated over some intervals in the pseudorapidities $\eta$ and $\eta^{\prime}$, reproduces the variance of $N_{\mathrm{ch}}$ defined by eq. (25).

Integrating this over the rapidity windows gives then

$$
\begin{aligned}
\sigma_{\mathrm{FF}}^{2} & =\left\langle N_{\mathrm{F}}\right\rangle+\frac{\sigma_{\mathrm{p}}^{2}}{\left\langle N_{\mathrm{p}}\right\rangle^{2}}\left\langle N_{\mathrm{F}}\right\rangle^{2} \\
\sigma_{\mathrm{FB}}^{2} & =\frac{\sigma_{\mathrm{p}}^{2}}{\left\langle N_{\mathrm{p}}\right\rangle^{2}}\left\langle N_{\mathrm{F}}\right\rangle\left\langle N_{\mathrm{B}}\right\rangle
\end{aligned}
$$

and similarly for the other interval combinations. This gives correlation coefficients

$$
R_{\mathrm{BF}}=\frac{1}{\sqrt{1+\frac{\left\langle N_{\mathrm{p}}\right\rangle^{2}}{\sigma_{\mathrm{p}}^{2}} \frac{1}{\left\langle N_{\mathrm{F}}\right\rangle}} \sqrt{1+\frac{\left\langle N_{\mathrm{p}}\right\rangle^{2}}{\sigma_{\mathrm{p}}^{2}} \frac{1}{\left\langle N_{\mathrm{B}}\right\rangle}}}
$$

\footnotetext{
${ }^{1}$ We are neglecting quantities that are higher order in the multiplicity fluctuations. For example one is approximating $\left\langle n_{i}^{2}\right\rangle \approx\left\langle n_{i}\right\rangle^{2}$ in the second term, since it is already proportional to the relative variance $\sigma_{\mathrm{p}}^{2} /\left\langle N_{\mathrm{p}}\right\rangle^{2}$ which can be assumed to be small.
} 
and correspondigly for $R_{\mathrm{FR}}$ and $R_{\mathrm{BF}}$.

To get a rough estimate of the numbers we shall neglect the $\eta$-dependence in eq. (26) and approximate $\tilde{n} \equiv \bar{n} / \sqrt{2 \pi \sigma_{\eta}} \approx 2$ (this is the charged multiplicity per unit rapidity and per participant). Taking the rapidity intervals $\delta$ and $\delta_{\mathrm{R}}$ for the different windows we then get

$$
\begin{aligned}
& R_{\mathrm{BF}}=\frac{1}{1+\frac{1}{\omega_{\mathrm{p}} \bar{n} \delta}} \\
& R_{\mathrm{FR}}=\frac{1}{\sqrt{1+\frac{1}{\omega_{\mathrm{p}} \tilde{n} \delta}} \sqrt{1+\frac{1}{\omega_{\mathrm{p}} \tilde{n} \delta_{\mathrm{R}}}}},
\end{aligned}
$$

where we have, following [36], denoted $\omega_{\mathrm{p}} \equiv \sigma_{\mathrm{p}}^{2} /\left\langle N_{\mathrm{p}}\right\rangle$. We then take from [36] the values $\omega_{\mathrm{p}} \approx 2.5$ for the $0-10 \%$ bin and $\omega_{\mathrm{p}} \approx 1.3$ for a midcentral $40-50 \%$. bin. This gives $R_{\mathrm{BF}} \approx 0.5$ and $R_{\mathrm{FR}} \approx 0.65$ for the more central bin and $R_{\mathrm{BF}} \approx 0.34$ and $R_{\mathrm{FR}} \approx 0.5$. These values of $R_{\mathrm{BF}}$ are the correlation coefficients quoted in [36]. As we have discussed, however, they do not correspond to the actual measured quantity.

Only at this point need we evoke the Gaussian approximation for the probability distribution. If we assume that the probability distribution is Gaussian, we can use eq. (19) to evaluate the $b$ parameter from the correlation coefficients (31) and (32) of the model. Putting the numbers estimated above into eq. (19) gives $b \approx 0.14$ for the central and $b \approx 0.12$ for the midcentral bin. These values are clearly far from the experimental result.

\subsection{Beyond the Gaussian approximation}

Although it seems unlikely, based on the result we just obtained, that this simple toy model would come near to explaining the experimental data, we shall still continue analysing it further in order to understand the effect of the Gaussian approximation. For this it is instructive to start over from the definition of the model as a probability distribution. We shall again neglect the $\eta$-dependence of the single particle spectrum to avoid encumbering our notations. The model is defined by a fluctuating number of participant nucleons $N_{\mathrm{p}}$, which gives the parameter for the Poisson-distributions of the measured multiplicities $N_{\mathrm{F}}, N_{\mathrm{B}}, N_{\mathrm{R}}$. We can therefore write down the probability distribution that characterizes the model as follows

$P\left(N_{\mathrm{p}}, N_{\mathrm{F}}, N_{\mathrm{B}}, N_{\mathrm{R}}\right)=P_{\mathrm{MCG}}\left(N_{\mathrm{p}}\right)\left(\frac{\left(\delta \tilde{n} N_{\mathrm{p}}\right)^{N_{\mathrm{F}}} e^{-\delta \tilde{n} N_{\mathrm{p}}}}{N_{\mathrm{F}} !}\right)\left(\frac{\left(\delta \tilde{n} N_{\mathrm{p}}\right)^{N_{\mathrm{B}}} e^{-\delta \tilde{n} N_{\mathrm{p}}}}{N_{\mathrm{B}} !}\right)\left(\frac{\left(\delta_{\mathrm{R}} \tilde{n} N_{\mathrm{p}}\right)^{N_{\mathrm{R}}} e^{-\delta_{\mathrm{R}} \tilde{n} N_{\mathrm{p}}}}{N_{\mathrm{R}} !}\right)$.

Here $P_{\mathrm{MCG}}\left(N_{\mathrm{p}}\right)$ is the probability distribution of events with different numbers of participant nucleons, presumably to be obtained from a Monte Carlo Glauber calculation. The first step in our approximation is to replace the Poissonian distributions by Gaussians in $N_{\mathrm{F}}, N_{\mathrm{B}}, N_{\mathrm{R}}$, which should be pretty safe as long as these multiplicities are all large enough 2 . We then obtain

$P\left(N_{\mathrm{p}}, N_{\mathrm{F}}, N_{\mathrm{B}}, N_{\mathrm{R}}\right)=\frac{P_{\mathrm{MCG}}\left(N_{\mathrm{p}}\right)}{\left(2 \pi \tilde{n} N_{\mathrm{p}}\right)^{3 / 2} \delta \sqrt{\delta_{\mathrm{R}}}} \exp \left[-\frac{\left(N_{\mathrm{F}}-\delta \tilde{n} N_{\mathrm{p}}\right)^{2}}{2 \delta \tilde{n} N_{\mathrm{p}}}-\frac{\left(N_{\mathrm{B}}-\delta \tilde{n} N_{\mathrm{p}}\right)^{2}}{2 \delta \tilde{n} N_{\mathrm{p}}}-\frac{\left(N_{\mathrm{R}}-\delta_{\mathrm{R}} \tilde{n} N_{\mathrm{p}}\right)^{2}}{2 \delta_{\mathrm{R}} \tilde{n} N_{\mathrm{p}}}\right]$.

\footnotetext{
${ }^{2}$ We are simultaneously approximating the discrete variables $N_{\mathrm{F}}, N_{\mathrm{B}}, N_{\mathrm{R}}$ by continuous ones
} 


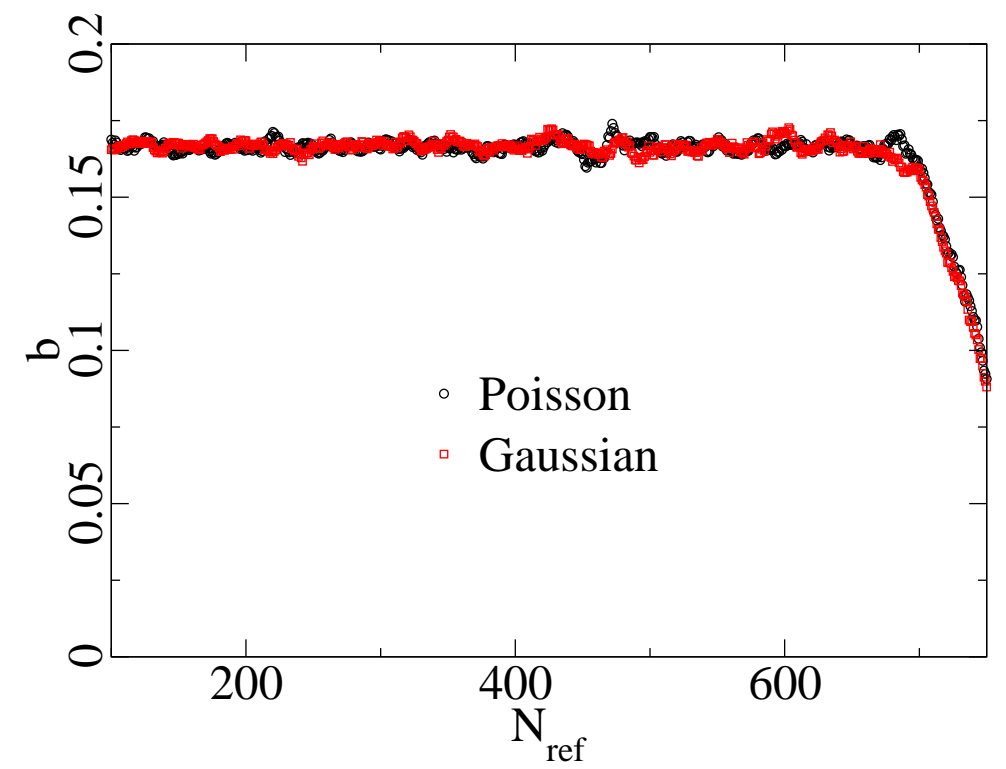

Figure 1: The correlation coefficient $b$ resulting from a direct Monte Carlo-Glauber evaluation of the probability distribution (33). Also shown is the result of the approximation where the Poisson distributions are replaced by Gaussians, but the distribution of $N_{\mathrm{p}}$ values is the same MCG one, i.e. eq. (34).

The next step in our approximation introduces a larger error. In the experimental analysis the centrality class is defined by events where the reference multiplicity $N_{\mathrm{R}}$ falls between a given lower and upper bound. Since the number of participants is not a directly experimentally observable quantity, it cannot be used as a selection criterion. We shall, however, assume that the centrality bin is defined by a Gaussian distribution of $N_{\mathrm{p}}$ with some variance $\sigma_{\mathrm{p}}^{2}$ :

$$
P_{\mathrm{MCG}}\left(N_{\mathrm{p}}\right) \approx \frac{1}{\sqrt{2 \pi \sigma_{\mathrm{p}}^{2}}} \exp \left[-\frac{\left(N_{\mathrm{p}}-\left\langle N_{\mathrm{p}}\right\rangle\right)^{2}}{2 \sigma_{\mathrm{p}}^{2}}\right] .
$$

The remaining approximation is to replace $N_{\mathrm{p}}$ by $\left\langle N_{\mathrm{p}}\right\rangle$ in the variances (but not the means!) of the Gaussians in $N_{\mathrm{F}}, N_{\mathrm{B}}, N_{\mathrm{R}}{ }^{3}$. This has the result of turning our probability distribution into a Gaussian in all four of its variables:

$$
P\left(N_{\mathrm{p}}, N_{\mathrm{F}}, N_{\mathrm{B}}, N_{\mathrm{R}}\right)=\frac{\exp \left[-\frac{\left(N_{\mathrm{p}}-\left\langle N_{\mathrm{p}}\right\rangle\right)^{2}}{2 \sigma_{\mathrm{p}}^{2}}-\frac{\left(N_{\mathrm{F}}-\delta \tilde{n} N_{\mathrm{p}}\right)^{2}}{2 \delta \tilde{n}\left\langle N_{\mathrm{p}}\right\rangle}-\frac{\left(N_{\mathrm{B}}-\delta \tilde{n} N_{\mathrm{p}}\right)^{2}}{2 \delta \tilde{n}\left\langle N_{\mathrm{p}}\right\rangle}-\frac{\left(N_{\mathrm{R}}-\delta_{\mathrm{R}} \tilde{n} N_{\mathrm{p}}\right)^{2}}{2 \delta_{\mathrm{R}} \tilde{n}\left\langle N_{\mathrm{p}}\right\rangle}\right]}{(2 \pi)^{2}\left(\tilde{n}\left\langle N_{\mathrm{p}}\right\rangle\right)^{3 / 2} \delta \sqrt{\delta_{\mathrm{R}}} \sigma_{\mathrm{p}}} .
$$

The remaining step is now to integrate eq. (36) over $N_{\mathrm{p}}$. Note that this integration is now done for fixed $N_{\mathrm{F}}, N_{\mathrm{B}}, N_{\mathrm{R}} \sqrt{4}$. If, as is typically the case for a realistic centrality bin, $\sigma_{\mathrm{p}}^{2}$ is relatively large

\footnotetext{
${ }^{3}$ This is equivalent to the approximation $\left\langle n_{i}^{2}\right\rangle \approx\left\langle n_{i}\right\rangle^{2}$ in the variance of $\mathrm{d} N / \mathrm{d} \eta$ mentioned in an earlier footnote.

${ }^{4}$ Note that since at no point do the experimentalists measure directly the impact parameter or $N_{\mathrm{p}}$, the correct procedure to get a distribution that corresponds to the measurement is to first integrate $P\left(N_{\mathrm{p}}, N_{\mathrm{F}}, N_{\mathrm{B}}, N_{\mathrm{R}}\right)$ over $N_{\mathrm{p}}$ to get the probability distribution $P\left(N_{\mathrm{F}}, N_{\mathrm{B}}, N_{\mathrm{R}}\right)$. From this one can then restrict to the conditional distribution $P\left(N_{\mathrm{F}}, N_{\mathrm{B}} \mid N_{\mathrm{R}}\right.$ fixed $)=P\left(N_{\mathrm{F}}, N_{\mathrm{B}}, N_{\mathrm{R}}\right) / P\left(N_{\mathrm{R}}\right)$ and compute $D_{f f}$ and $D_{f b}$. This is not the same thing as computing $D_{f f}$ and $D_{f b}$ for a fixed impact parameter or $N_{\mathrm{p}}$ and then averaging the results over $N_{\mathrm{p}}$; which would imply that $N_{\mathrm{p}}$ is actually measured event-by event.
} 
and $\delta_{\mathrm{R}} \gg \delta$, this integral is dominated by the reference multiplicity term $\left(N_{\mathrm{R}}-\delta_{\mathrm{R}} \tilde{n} N_{\mathrm{p}}\right)^{2}$. This means that selecting events with a fixed $N_{\mathrm{R}}$ selects events with $N_{\mathrm{p}}$ close to $N_{\mathrm{R}} /\left(\delta_{\mathrm{R}} \tilde{n}\right)$, not the typical value $\left\langle N_{\mathrm{p}}\right\rangle$ of the original set of events. This causes the probability distribution of $N_{\mathrm{F}}$ and $N_{\mathrm{B}}$ to be peaked around $\left(\delta / \delta_{\mathrm{R}}\right) N_{\mathrm{R}}$ and thus induces a correlation between $N_{\mathrm{F}}, N_{\mathrm{B}}, N_{\mathrm{R}}$. An easy way to perform the $N_{\mathrm{p}}$-integration is to realize that the result is a Gaussian in $N_{\mathrm{F}}, N_{\mathrm{B}}, N_{\mathrm{R}}$. To obtain the coefficients of the Gaussian in $N_{\mathrm{F}}, N_{\mathrm{B}}, N_{\mathrm{R}}$ it is then sufficient to evaluate the expectation values and the covariance matrix, which can also be done by integrating first over the multiplicities and only then over $N_{\mathrm{p}}$. In any case, from eq. (36) one obtains

$$
\begin{aligned}
\left\langle N_{\mathrm{F}}\right\rangle & =\left\langle N_{\mathrm{B}}\right\rangle=\delta \tilde{n}\left\langle N_{\mathrm{p}}\right\rangle \\
\left\langle N_{\mathrm{R}}\right\rangle & =\delta_{\mathrm{R}} \tilde{n}\left\langle N_{\mathrm{p}}\right\rangle \\
\left\langle N_{\mathrm{F}}{ }^{2}\right\rangle-\left\langle N_{\mathrm{F}}\right\rangle^{2} & =\delta \tilde{n}\left\langle N_{\mathrm{p}}\right\rangle+(\delta \tilde{n})^{2} \sigma_{\mathrm{p}}^{2}=\left\langle N_{\mathrm{F}}\right\rangle+\frac{\sigma_{\mathrm{p}}^{2}}{\left\langle N_{\mathrm{p}}\right\rangle^{2}}\left\langle N_{\mathrm{F}}\right\rangle^{2} \\
\left\langle N_{\mathrm{F}} N_{\mathrm{B}}\right\rangle-\left\langle N_{\mathrm{F}}\right\rangle\left\langle N_{\mathrm{B}}\right\rangle & =(\delta \tilde{n})^{2} \sigma_{\mathrm{p}}^{2}=\frac{\sigma_{\mathrm{p}}^{2}}{\left\langle N_{\mathrm{p}}\right\rangle^{2}}\left\langle N_{\mathrm{F}}\right\rangle\left\langle N_{\mathrm{B}}\right\rangle
\end{aligned}
$$

and correspondingly for the other combinations of $\mathrm{F}, \mathrm{B}, \mathrm{R}$. We have how rederived the formulas used in eqs. (28) and (29) to evaluate the result of the model of [36] in the Gaussian approximation previously.

In order to evaluate the importance of the different approximations we have also used a simple Monte Carlo Glauber implementation to evaluate the probability distribution (33). The details of our simple Monte Carlo Glauber model are described in Appendix $\mathrm{A}$ and the result of the computation is shown in fig. 1. It turns out that the result is very close to $b=\delta /\left(\delta+\delta_{\mathrm{R}}\right)=1 / 6$. This corresponds to the limit $\omega_{\mathrm{p}} \rightarrow \infty$ in the results eqs. (31) and (32) of the toy model. This is easy to understand a posteriori. In the Gaussian approximation we assumed that one is only looking at events in a centrality bin that is defined by a relatively compact distribution in $N_{\mathrm{p}}$. This is in fact not the case; the centrality bin is defined using the reference multiplicity, and the distribution of impact parameters $P_{\mathrm{MCG}}\left(N_{\mathrm{p}}\right)$ in eq. (33) includes the whole "min bias" distribution of impact parameters, and is therefore very wide. The fact that one must take the limit $\sigma_{\mathrm{p}}^{2} \rightarrow \infty$ means, in the language of Sec. 3. that the correlation coefficients are all approaching unity. They do so, however, in such a way that $b$ approaches $\delta /\left(\delta+\delta_{\mathrm{R}}\right)$. This is in some sense the natural upper limit for $b$, if the correlation function eq. (3) consists of only a Poissonian and long range piece; and the most realistic way of modifying it is to introduce a short range correlation which increases the fluctuations in the reference multiplicity. We shall now turn to a more general parametrization where this can be seen more explicitly.

\section{The effect of intrinsic correlations}

Let us then construct a simple parametrization of long range correlation effects in addition to the impact parameter fluctuations. As we saw in the previous section, assuming a Gaussian distribution for the impact parameter fluctuations (i.e. neglecting the fact that the centrality selection is done using $N_{\mathrm{R}}$ ) is not a very good approximation. We would therefore like to construct a model where the impact parameter fluctuations are parametrized by an $N_{\mathrm{p}}$ drawn from a Monte Carlo Glauber calculation, and particle production for a fixed $N_{\mathrm{p}}$ then includes physical long range rapidity correlations. We emphasize that we are not assuming that the physical mechanism of 


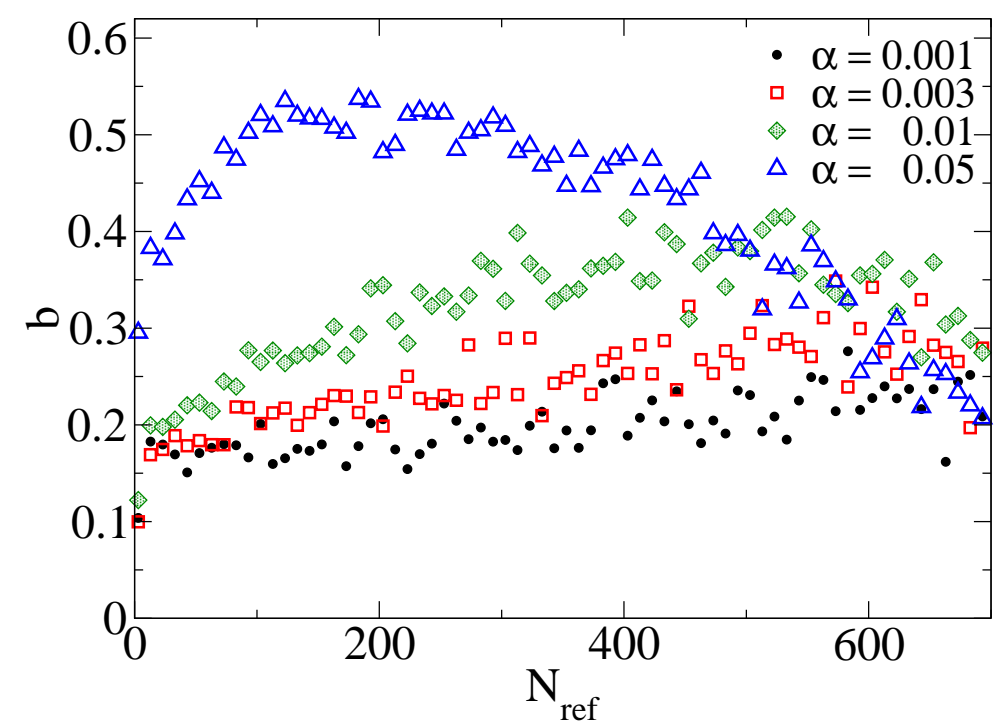

Figure 2: The constrained correlation coefficient $b$ as a function of $N_{\mathrm{R}}$ for $K=0.001$ and different values of $\alpha$.

particle production could be decomposed into independent production from participant nucleons. We use the quantity $N_{\mathrm{p}}$ as a convenient proxy for the impact parameter dependent overlap area of the colliding nuclei and its fluctuations. The main reason for using $N_{\mathrm{p}}$ instead of the overlap area is its easy implementation in our simple Monte Carlo Glauber code.

We will assume that the probability distribution for a fixed $N_{\mathrm{p}}$ is Gaussian:

$$
P\left(N_{\mathrm{p}}, N_{\mathrm{F}}, N_{\mathrm{B}}, N_{\mathrm{R}}\right)=P_{\mathrm{MCG}}\left(N_{\mathrm{p}}\right) \frac{1}{(2 \pi)^{3 / 2} \operatorname{det} \Sigma\left(N_{\mathrm{p}}\right)} \exp \left[-\frac{1}{2} \Delta_{U} \Sigma_{U V}^{-1}\left(N_{\mathrm{p}}\right) \Delta_{V}\right],
$$

where now $\Delta_{U}=N_{U}-\bar{N}_{U}\left(N_{\mathrm{p}}\right)$ (we denote the expectation value for a fixed $N_{\mathrm{p}}$ by $\bar{N}_{U}\left(N_{\mathrm{p}}\right)$ to separate it from the measured expectation value $\left\langle N_{U}\right\rangle$ which is averaged over some range of impact parameters). The expectation values for a fixed $N_{\mathrm{p}}$ are

$$
\begin{aligned}
\bar{N}_{\mathrm{F}}=\bar{N}_{\mathrm{B}} \equiv \bar{N} & =\delta \tilde{n} N_{\mathrm{p}} \\
\bar{N}_{\mathrm{R}} & =\delta_{\mathrm{R}} \tilde{n} N_{\mathrm{p}}
\end{aligned}
$$

and also the correlation matrix for fixed impact parameters depends on $N_{\mathrm{p}}$ through the expectation values $\bar{N}_{U}$ as

$$
\Sigma\left(N_{\mathrm{p}}\right)=\frac{1}{\operatorname{det} \Sigma}\left(\begin{array}{ccc}
\bar{N}_{\mathrm{F}}+(K+\alpha) \bar{N}^{2} & K \bar{N}^{2} & K \bar{N} \bar{N}_{\mathrm{R}} \\
K \bar{N}^{2} & \bar{N}+(K+\alpha) \bar{N}^{2} & K \bar{N} \bar{N}_{\mathrm{R}} \\
K \bar{N} \bar{N}_{\mathrm{R}} & K \bar{N} \bar{N}_{\mathrm{R}} & \bar{N}_{\mathrm{R}}+(K+\alpha) \bar{N}_{\mathrm{R}}{ }^{2}
\end{array}\right) .
$$

Here we have introduced two additional parameters: $\alpha$ describes the increased fluctuations of the multiplicity due to a short range rapidity correlation, and $K$ represents a dynamical long range fluctuation that increases both the local fluctuations $\Sigma_{\mathrm{FF}}, \ldots$ and generates a correlation 

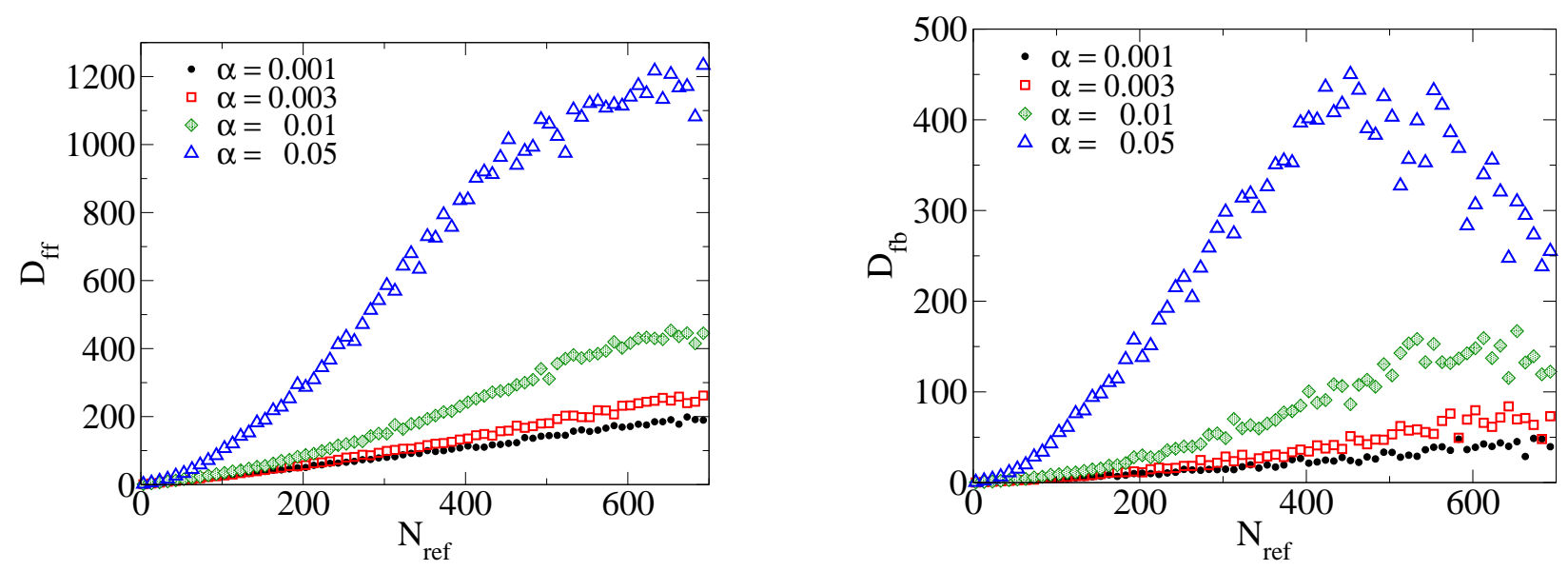

Figure 3: The constrained variance $D_{f f}$ and covariance $D_{b f}$ with the same values for $\alpha$ and $K=0.001$ as in fig. 2 .

between the different rapidity windows $\Sigma_{\mathrm{FR}}, \ldots$ In terms of the rapidity correlation function these correspond to a parametrization

$$
\left.C\left(\eta, \eta^{\prime}\right)\right|_{N_{\mathrm{p}} \text { fixed }} \equiv \delta\left(\eta-\eta^{\prime}\right)\left\langle\frac{\mathrm{d} N}{\mathrm{~d} \eta}\right\rangle+\left[K+\alpha \theta\left(Y-\left|\eta-\eta^{\prime}\right|\right)\right]\left\langle\frac{\mathrm{d} N}{\mathrm{~d} \eta}\right\rangle\left\langle\frac{\mathrm{d} N}{\mathrm{~d} \eta^{\prime}}\right\rangle,
$$

where $Y$ is a characteristic scale of short range rapidity correlations that we assume is larger than the size of the rapidity windows but less than the separation between different rapidity window: 5 . Our parametrization reduces to the model of Ref. [36] in the limit $\alpha=K=0$. The natural scales at which the values of $\alpha$ and $K$ can vary can be estimated from the relation to the parameter $k$ of the negative binomial distribution of multiplicities. It can be seen from eq. (5) that for relatively small rapidity intervals $1 / k=\alpha+K$ (recall that this is true for a fixed impact parameter, or fixed $N_{\mathrm{p}}$ in our parametrization). For central collisions the PHENIX experiment [43] $k \sim 690$ for central collisions, so typically we would expect $\alpha, K \sim 0.001$.

We can then evaluate the correlation using a simple Monte Carlo Glauber implementation described in Appendix A. Our results are summarized in figs. 2, 3, 4, Figure 2 shows the dependence of $b$ on $\alpha$; the strength of the short range rapidity correlation. We see that increasing $\alpha$ increases the correlation $b$. This is to be understood in the following way: the main effect of increased short range correlations $\alpha$ is to increase the fluctuations (uncorrelated with $N_{\mathrm{F}}, N_{\mathrm{B}}$ ) in $N_{\mathrm{R}}$. These increased fluctuations then decrease the correlation between the reference and the F,B multiplicities. As can be seen from eq. (19), this has the effect of increasing $b$. For a very large $\alpha \sim 0.05$ one can even reach $b \sim 0.5$, but as can be seen from fig. 3 the corresponding values of $D_{f f}, D_{f b}$ become much larger than the experimental values. Increasing $\alpha$ increases the ratio $b=D_{f b} / D_{f f}$ closer to the experimental result, but it also increases the fluctuations in $N_{\mathrm{F}}$ and $N_{\mathrm{B}}$ separately beyond what is observed experimentally.

Figure 4 shows the dependence of the correlation on the parameter $K$ describing the dynamical long distance rapidity correlations. It is seen that both $D_{f f}$ and $D_{f b}$ and therefore also $b$ are,

\footnotetext{
${ }^{5}$ We are neglecting the fact that in the actual STAR results the reference window is often wider than its distance from the F,B windows. Taking this into account folly would unneccessarily copmplicate the parametrization (44) without changing our results much.
} 


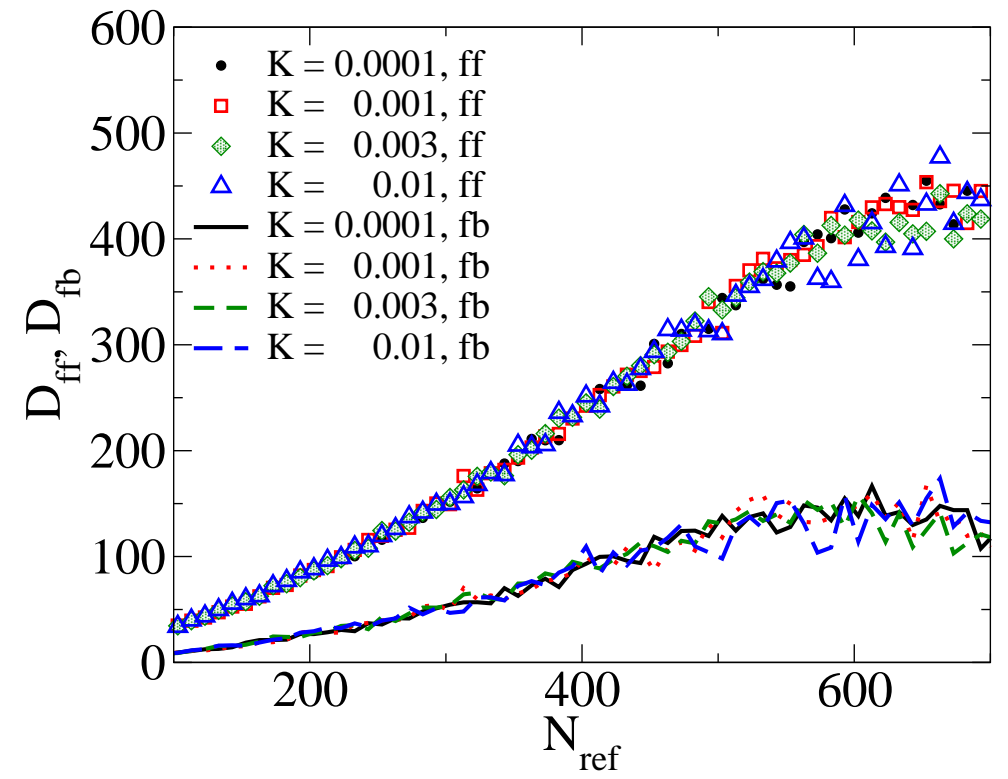

Figure 4: The constrained variance $D_{f f}$ and covariance $D_{b f}$ for $\alpha=0.01$ and different values of $K$.

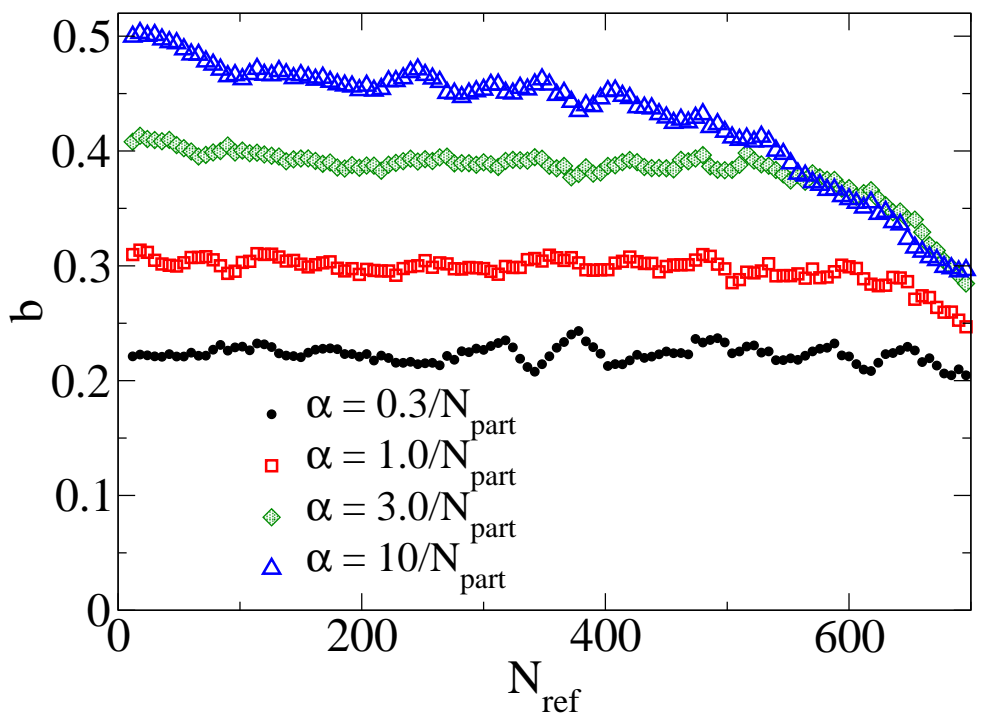

Figure 5: The Monte Carlo result for $b$ with $\alpha$ and $K$ depending on centrality as $K=1 / N_{\mathrm{p}}$ and $\alpha \sim 1 / N_{\mathrm{p}}$ with a coefficient shown in the figure. 
at the statistical accuracy of our Monte Carlo calculation, independent of $K$. The most natural explanation for this is that, as observed previously, the impact parameter fluctuations already generate a correlation that corresponds to the limit $\sigma_{\mathrm{p}}^{2} \rightarrow \infty$, or a maximal FB correlation. Adding a small physical dynamical correlation described by a reasonable $K$ is negligible compared to this. This leads to a significant observation concerning the experimental analysis technique, namely that it would be better if the centrality selection could be done, into as narrow bins as possible, using another observable as independent as possible from $N_{\mathrm{R}}$. An example of this effect is the STAR observation (fig. 1(a) of Ref. [1]) that determining the centrality using the ZDC instead of $N_{\mathrm{R}}$ leads to a smaller measured $b$.

In our simple parametrization we have so far completely neglected the dependence of the parameters on centrality. The charged multiplicity per participant $\tilde{n}$ varies among centralities and, more importantly, the dynamical correlation strengths $\alpha, K$ should to a first approximation scale with the inverse transverse overlap area, or $\sim 1 / N_{\mathrm{p}}$. Figure [5] shows the result for $b$ when this kind of a scaling is taken into account.

The purpose of this paper is to stress the qualitative effect of taking into account the correlation with the reference multiplicity, not to perform a detailed fit to experimental data. A further finetuning of the centrality dependence of the parameters would not change the features we are addressing here.

\section{Conclusions}

The STAR measurements of long range rapidity correlations point to a very intriguing picture of strong correlations from the initial strong color fields in the initial stages of the collision. In spite of the apparent simplicity of the experimental observable (counting charged particles in a relatively large region of the detector), the measurements turn out to be challenging to interpret. The experimental analysis is done by treating separately events with different reference multiplicities. Thus for a consistent treatment one must consider on equal footing also the correlations with the reference rapidity window. This turns the problem from a 2-variable into a 3 -variable correlation, which has not always been fully appreciated in the literature. The values quoted by the STAR collaboration are for a forward-backward correlation for a fixed reference multiplicity. Since the result shows that for central collisions there is a strong correlation between the forward and backward multiplicities it would be unphysical to neglect the correlation with the reference multiplicity.

We have in this paper discussed long range rapidity correlations in the charged particle multiplicity in terms of only very general assumptions on multiplicity correlations. In a Gaussian approximation for the probability distribution of events in a centrality class, we find an upper limit $b<1 / 2$ for the conditional correlation when $\delta<\delta_{\mathrm{R}}$ and the $\mathrm{F}$ and $\mathrm{B}$ windows are far from each other in rapidity. We then give up the Gaussian approximation and construct a simple parametrization of the long and short range correlation, including the effects of impact parameter fluctuations with a simple Monte Carlo Glauber model. We show that impact parameter fluctuations alone are not sufficient to explain the observed data. Because of the correlation with the reference multiplicity, the measured conditional correlation coefficient $b$ turns out not to be very sensitive to the strength of the dynamical long range correlation. It does depend strongly on the short range rapidity correlation through its effect on the fluctuations of the reference multiplicity. Nevertheless we do not find a parametrization that would agree with the large $b$ reported by the STAR experiment. More experimental data would be welcome to disentangle the interplay between 
impact parameter, short range correlations leading to increased fluctuations and genuine long range correlations. Having a wide enough rapidity between the measured windows to actually see the decrease of the long range correlation (as opposed to the geometrical ones that are truly infinite range), would be useful but might not be possible within the STAR TPC. The dependence of the correlation on the sizes of the rapidity windows $\left(\delta\right.$ and $\left.\delta_{\mathrm{R}}\right)$ could be revealing. Preliminary STAR data indicates that $b$ increases when $\delta$ grows, for a fixed $\delta_{\mathrm{R}}$, which is in agreement with our qualitative expectations based on the discussion in sec. 4 Also consistency between the STAR [1] and PHENIX [41, 43] measurements is yet not established, as they have both a different experimental coverage and analysis method.

\section{Acknowledgements}

We are grateful to B.K. Srivastava for patiently taking the time to answer our naive questions and take seriously our suggestions for alteranative ways of plotting the data. We gratefully acknowledge conversations and correspondence with G. Torrieri, A. Bzdak, J.-Y. Ollitrault, K. Fukushima, R. Venugopalan and F. Gelis. L.M. thanks the hospitality of CEA-Saclay where this work was initiated as a result of critical and insightful comments by J.-Y. Ollitrault. The research of L.M. is supported under DOE Contract No. DE-AC02-98CH10886. T.L. is supported by the Academy of Finland, project 126604.

\section{A. MC Glauber implementation}

We do not use an established Monte Carlo Glauber code, but a very simple implementation of our own. For each configuration we first generate two configurations of nucleons according to a Woods-Saxon distribution with $R_{\mathrm{A}}=6.38 \mathrm{fm}$ and surface diffuseness $d=0.535 \mathrm{fm}$. We then draw randomly an impact parameter vector $\boldsymbol{b}_{\perp}$. If a nucleon is at a transverse distance of less than $\sqrt{\sigma_{N N} / \pi}$ with $\sigma_{N N}=41 \mathrm{mb}$ of a nucleon in the other nucleus, it is considered as a participant.

Once we have a value $N_{\mathrm{p}}$, one then has to generate random variables according to the distribution eq. (44). For this purpose one must diagonalize the correlation matrix (44). This is effectively done in the following way. One generates 3 independent Gaussian random numbers $\xi, \xi_{ \pm}$with zero mean and the variances

$$
\begin{aligned}
\left\langle\xi^{2}\right\rangle & =1 \\
\left\langle\xi_{ \pm}^{2}\right\rangle & =1 \pm \frac{K \bar{N} \bar{N}_{\mathrm{R}}}{\sqrt{\frac{1}{2}\left(\bar{N}+\alpha \bar{N}^{2}\right)+K \bar{N}^{2}} \sqrt{\bar{N}_{\mathrm{R}}+(\alpha+K) \bar{N}_{\mathrm{R}}^{2}}} .
\end{aligned}
$$

One then solves $\Delta_{\mathrm{F}, \mathrm{B}, \mathrm{R}}$ from

$$
\begin{aligned}
\Delta_{\mathrm{F}}-\Delta_{\mathrm{B}} & =\sqrt{2} \sqrt{\bar{N}+\alpha \bar{N}^{2}} \xi \\
\Delta_{\mathrm{F}}+\Delta_{\mathrm{B}} & =\sqrt{\bar{N}+(\alpha+2 K) \bar{N}^{2}}\left(\xi_{+}+\xi_{-}\right) \\
\Delta_{R} & =\frac{1}{\sqrt{2}} \sqrt{\bar{N}_{\mathrm{R}}+(\alpha+K) \bar{N}_{\mathrm{R}}^{2}}\left(\xi_{+}-\xi_{-}\right) .
\end{aligned}
$$

It is straightforward to verify that this procedure gives variables $N_{\mathrm{F}}, N_{\mathrm{B}}, N_{\mathrm{R}}$ with the desired correlations, eq. (44). 


\section{References}

[1] STAR, B. I. Abelev et al., arXiv:0905.0237 [nucl-ex]

[2] T. J. Tarnowsky, arXiv:0807.1941 [nucl-ex]

[3] L. D. McLerran and R. Venugopalan, Phys. Rev. D49, 2233 (1994), arXiv:hep-ph/9309289.

[4] L. D. McLerran and R. Venugopalan, Phys. Rev. D49, 3352 (1994), arXiv:hep-ph/9311205.

[5] Y. V. Kovchegov, Phys. Rev. D54, 5463 (1996) | arXiv:hep-ph/9605446].

[6] A. Kovner, L. D. McLerran and H. Weigert, Phys. Rev. D52, 6231 (1995), arXiv:hep-ph/9502289.

[7] A. Kovner, L. D. McLerran and H. Weigert, Phys. Rev. D52, 3809 (1995) arXiv:hep-ph/9505320.

[8] J. Jalilian-Marian, A. Kovner, L. D. McLerran and H. Weigert, Phys. Rev. D55, 5414 (1997), arXiv:hep-ph/9606337.

[9] J. Jalilian-Marian, A. Kovner, A. Leonidov and H. Weigert, Nucl. Phys. B504, 415 (1997), arXiv:hep-ph/9701284.

[10] J. Jalilian-Marian, A. Kovner, A. Leonidov and H. Weigert, Phys. Rev. D59, 014014 (1999), arXiv:hep-ph/9706377.

[11] E. Iancu, A. Leonidov and L. D. McLerran, Nucl. Phys. A692, 583 (2001), arXiv:hep-ph/0011241].

[12] E. Iancu, A. Leonidov and L. D. McLerran, Phys. Lett. B510, 133 (2001), arXiv:hep-ph/0102009.

[13] E. Ferreiro, E. Iancu, A. Leonidov and L. McLerran, Nucl. Phys. A703, 489 (2002), |arXiv:hep-ph/0109115.

[14] A. Krasnitz and R. Venugopalan, Phys. Rev. Lett. 84, 4309 (2000), arXiv:hep-ph/9909203.

[15] A. Krasnitz and R. Venugopalan, Nucl. Phys. B557, 237 (1999), arXiv:hep-ph/9809433.

[16] T. Lappi, Phys. Rev. C67, 054903 (2003), arXiv:hep-ph/0303076].

[17] T. Lappi and L. McLerran, Nucl. Phys. A772, 200 (2006) | $\operatorname{arXiv:hep-ph/0602189].~}$

[18] Y. V. Kovchegov, E. Levin and L. D. McLerran, Phys. Rev. C63, 024903 (2001), arXiv:hep-ph/9912367].

[19] N. Armesto, L. McLerran and C. Pajares, Nucl. Phys. A781, 201 (2007), arXiv:hep-ph/0607345|.

[20] K. Fukushima and Y. Hidaka, Nucl. Phys. A813, 171 (2008), arXiv:0806.2143 [hep-ph]].

[21] N. Armesto, M. A. Braun, E. G. Ferreiro and C. Pajares, Phys. Rev. Lett. 77, 3736 (1996), arXiv:hep-ph/9607239.

[22] M. A. Braun, C. Pajares and J. Ranft, Int. J. Mod. Phys. A14, 2689 (1999), arXiv:hep-ph/9707363.

[23] L. Cunqueiro, E. G. Ferreiro and C. Pajares, PoS CFRNC2006, 019 (2006), arXiv:hep-ph/0611034].

[24] STAR, J. Adams et al., Phys. Rev. C73, 064907 (2006), arXiv:nucl-ex/0411003.

[25] STAR, J. Adams et al., Phys. Rev. C72, 044902 (2005), arXiv:nucl-ex/0504031.

[26] STAR, J. Adams et al., J. Phys. G32, L37 (2006), |arXiv:nucl-ex/0509030].

[27] J. Putschke, J. Phys. G34, S679 (2007), arXiv:nucl-ex/0701074].

[28] STAR, M. Daugherity, J. Phys. G35, 104090 (2008), arXiv:0806.2121 [nucl-ex]].

[29] PHENIX, A. Adare et al., Phys. Rev. C78, 014901 (2008), |arXiv:0801.4545 [nucl-ex]].

[30] M. P. McCumber and f. t. P. Collaboration, J. Phys. G35, 104081 (2008), [arXiv:0804.4319 [nucl-ex]].

[31] PHOBOS, B. Alver et al., J. Phys. G35, 104080 (2008), |arXiv:0804.3038 [nucl-ex]].

[32] A. Dumitru, F. Gelis, L. McLerran and R. Venugopalan, Nucl. Phys. A810, 91 (2008), arXiv:0804.3858 [hep-ph].

[33] S. Gavin, L. McLerran and G. Moschelli, Phys. Rev. C79, 051902 (2009), arXiv:0806.4718 [nucl-th]].

[34] K. Dusling, D. Fernandez-Fraile and R. Venugopalan, Nucl. Phys. A828, 161 (2009). arXiv:0902.4435 [nucl-th].

[35] F. Gelis, T. Lappi and L. McLerran, Nucl. Phys. A828, 149 (2009), arXiv:0905.3234 [hep-ph].

[36] V. P. Konchakovski, M. Hauer, G. Torrieri, M. I. Gorenstein and E. L. Bratkovskaya, Phys. Rev. C79, 034910 (2009), arXiv:0812.3967 [nucl-th]].

[37] A. Bzdak, Phys. Rev. C80, 024906 (2009), [arXiv:0902.2639 [hep-ph].

[38] A. Bzdak, arXiv:0904.0869 [hep-ph]

[39] A. Bzdak, arXiv:0906.2858 [hep-ph]

[40] A. Giovannini and L. Van Hove, Z. Phys. C30, 391 (1986)

[41] PHENIX, S. S. Adler et al., Phys. Rev. C76, 034903 (2007), arXiv:0704.2894 [nucl-ex].

[42] STAR, B. K. Srivastava, Int. J. Mod. Phys. E16, 3371 (2007), arXiv:nucl-ex/0702054].

[43] PHENIX, A. Adare et al., Phys. Rev. C78, 044902 (2008) , arXiv:0805.1521 [nucl-ex]]. 